\title{
Apoyo social percibido e implicación escolar: Correlaciones y variabilidad
}

\author{
Iker Izar de la Fuente Díaz de Cerio, Arántzazu Rodríguez Fernández y \\ Naiara Escalante Mateos \\ Universidad del País Vasco/EHU (España)
}

\begin{abstract}
El apoyo social percibido es crucial en el comportamiento escolar de los adolescentes, especialmente en la implicación escolar. En general, los contextos cercanos de una persona promueven su implicación escolar, pero no todas las fuentes de apoyo social tienen el mismo efecto. Los objetivos de esta investigación son dos: (a) analizar las relaciones entre el apoyo social percibido y la implicación escolar; e (b) identificar la variabilidad del apoyo social percibido y la implicación escolar en razón del sexo y nivel académico de los progenitores. Participaron en la investigación 323 estudiantes de Educación Secundaria de un instituto de Vitoria-Gasteiz con edades comprendidas entre los 13 y 18 años $(M=14.41 ; D T=1.18)$, de los cuales 128 eran chicos (40\%) y $195(60 \%)$ chicas, a los que se aplicaron los cuestionarios: APIK (apoyo social) y SEM (implicación escolar). Los resultados certifican que el apoyo social percibido correlaciona con la implicación escolar, predominando el apoyo del profesorado. Respecto a la variabilidad en sexo y nivel académico de los progenitores únicamente se han encontrado diferencias significativas en el apoyo social percibido de las amistades a favor de las mujeres.
\end{abstract}

Palabras clave: Apoyo social percibido, implicación escolar, correlaciones, sexo, nivel educativo de progenitores.

Perceived social support and school engagement: Correlations and variability. Perceived social support is crucial in the adolescent school behaviour, especially in school engagement. In general, a person's close contexts promote his school engagement, but not all the sources of social support have the same effect. The objectives of this investigation are two: on the one hand (a) analyse the relation between perceived social support and school involvement; and on the other hand (b) identify the variability of perceived social support and school engagement based on sex and parents' educational level. 323 students of Secondary Education of a high school of Vitoria-Gasteiz aged between 12 to 18 years old $(M=14.41 ; D T=1.18)$ participated in the study, being 128 of them boys $(40 \%)$ and 195 girls $(60 \%)$. The questionnaires APIK (social support) and SEM (school engagement) were used. The obtained results prove that perceived social support correlates with school engagement, being the support from the teachers the one with the highest punctuations. According to the variability based on sex and parents' educational level of the second objective, there are only differences in perceived social support, being them in favour of women.

Keywords: Perceived social support, school engagement, correlations, sex, parents' educational level.

Correspondencia: Iker Izar de la Fuente Díaz de Cerio. Universidad del País Vasco Universidad del País Vasco (UPV/EHU). C/ Juan Ibáñez de Sto. Domingo, nº1. C.P.: 01006. Vitoria-Gasteiz, Álava (España). E-mail: iker.izardelafuente@ehu.eus 


\section{El apoyo social}

Durante los últimos años, el apoyo social se ha convertido en un tema de gran interés en distintas disciplinas científicas (Alemán, 2013), convirtiéndose en uno de los más estudiados en los últimos años del siglo XX (Gracia, Herrero, y Musitu, 2002). En consecuencia, se ha generado una gran diversidad conceptual con maneras muy diferentes de entenderlo y, por lo tanto, de evaluarlo (Azpiazu, 2016).

Son las innumerables formas de definir el apoyo social que se han creado desde la década de los setenta (Barrón, 1996) cuando Cassel (1974a, 1974b), Cobb (1976) y Caplan (1974) consolidaron el constructo como objeto de estudio las que hacen que en la actualidad no exista una definición clara y consensuada del mismo (Barrón y Sánchez, 2001). De cualquier modo, como punto de partida, se puede entender de manera genérica como el conjunto de recursos proporcionados entre las personas (Cohen, Mermelstein, Kamarck, y Hoberman, 1985). Este constituye un proceso dinámico que evoluciona y va cambiando mediante las relaciones establecidas (Alemán, 2013), lo que lo convierte en un metaconcepto multidimensional (Wilcox y Vernberg, 1985) y multinivel (Barrón y Sánchez, 2001).

De todas las formas de entender el constructo, hay dos definiciones que posiblemente sean las más utilizadas: la realizada por Tardy (1985) y la desarrollada por Lin (1986). Tardy (1985) diferenció cinco dimensiones ordenadas jerárquicamente: dirección, disposición, descripción/evaluación, contenido y redes de apoyo. La segunda definición, considerada una de las más completas (Azpiazu, 2016; Barrón y Sánchez, 2001; Ramos, 2015), entiende el apoyo social como las provisiones instrumentales y/o expresivas -percibidas o reales- aportadas por la comunidad, redes sociales y personas de confianza, pudiendo darse tanto en situaciones cotidianas como de crisis (Lin, 1986).

\section{Relación del apoyo social y la implicación escolar}

Se ha demostrado que el apoyo social es crucial en el comportamiento escolar de los adolescentes (Rodríguez, Ramos, Ros, Fernández, y Revuelta, 2016), especialmente en la implicación escolar (Rodríguez, Ramos, Ros, y Zuazagoitia, 2018). Tradicionalmente la implicación se ha identificado como una participación activa, (Fredricks, Blumenfeld, y Paris, 2004) considerándose un elemento clave para el éxito académico (Ros, Goikoetxea, Gairín, y Lekue, 2012). En el contexto escolar, se define como un proceso psicológico que implica esfuerzo, inversión, atención e interés por parte del alumnado (Marks, 2000). Aunque todavía no existe concreción conceptual unívoca sobre la implicación (Veiga, Burden, Appleton, Taveira, y Galvao, 2014), se ha admitido su multidimensionalidad (Lewis, Huebner, Malone, y Valois, 2011; Ramos, 2015) aceptándose de manera generalizada el modelo tridimensional (Ros y Zuazagoitia, 2015). De este modo, el planteamiento de Fredricks, Blumenfeld, y Paris (2004) que diferencia entre implicación cognitiva, emocional y conductual ha sido el más reconocido. 
La dimensión conductual se entiende como el comportamiento observable de los estudiantes mediante el que se manifiesta su relación con la escuela y el aprendizaje: la asistencia a clase, la atención prestada, el esfuerzo, etc. (Ros y Zuazagoitia, 2015; Voelkl, 2012; Wang y Holcombe, 2010). La dimensión emocional se refiere a la conexión del estudiante con la escuela, a su interés, valores y sentimientos hacia esta (Furrer y Skinner, 2003). Por último, la cognitiva hace referencia a la implicación psicológica del alumnado en el aprendizaje, al conocimiento y habilidades así como a la motivación interna por aprender (Ros y Zuazagoitia, 2015).

En general, los contextos cercanos de una persona promueven su implicación escolar (Roeser, Eccles, y Sameroff, 2000), pero no todas las fuentes de apoyo social (familia, iguales y profesores) tienen el mismo efecto (Wang y Eccles, 2012). Pese a ello, el apoyo por parte de la familia (Christenson y Thurlow, 2004), de los iguales (Perdue, Manzeske, y Estell, 2009) y del profesorado (Roorda, Koomen, Spilt, y Oort, 2011) ha mostrado correlaciones con la implicación escolar. Hay estudios que han demostrado que el apoyo del profesorado es la fuente más fuertemente asociada a la implicación escolar seguida por el apoyo familiar, mostrando ambas la asociación más intensa con la implicación emocional. Respecto al apoyo de los amigos, únicamente correlaciona con la implicación conductual y emocional, siendo la relación con esta última, de nuevo, la de mayor intensidad (Ramos, 2015).

Variabilidad del apoyo social: sexo y nivel educativo de progenitores

Las diferentes formas de relacionarse de mujeres y hombres están estrechamente relacionadas con el apoyo social (Azpiazu, 2016). Las investigaciones indican que son las mujeres las que perciben mayor apoyo social (Demaray y Malecki, 2002), pero si se realizan los análisis centrándose en cada fuente de apoyo, los resultados difieren levemente. En el apoyo familiar, los estudios o no encuentran diferencias entre hombres y mujeres (Musitu y Cava, 2003), o lo hacen en favor de los hombres (Cheng y Chan, 2004). En el apoyo de las amistades, por el contrario, parece no haber discrepancias en que las mujeres perciben mayor apoyo de sus iguales (Malecki y Demaray, 2003; Musitu y Cava, 2003). Por último, en el apoyo del profesorado, algunos estudios defienden que no existe variabilidad alguna respecto al sexo (Malecki y Demaray, 2003), mientras que otros si encuentran diferencias a favor de las mujeres (Reddy, Rhodes, y Mulhall, 2003).

El nivel académico de los progenitores es una variable que se ha comenzado a analizar asociada al apoyo social en investigaciones relacionadas con distintos ámbitos educativos, encontrándose que el apoyo familiar se ve afectado de manera significativa por el nivel educativo de los progenitores (Bazán, Sánchez, y Castañeda, 2007), incluso en bebés (Mangrio, Hansen, Lindstrom, Kohler, y Rosvall, 2011). No obstante, en estos 
estudios se han identificado carencias metodológicas por lo que se cuestiona dicha relación entre variables.

Variabilidad de la implicación escolar: sexo y nivel educativo de progenitores

Las investigaciones en torno a la variabilidad de la implicación escolar respecto al sexo no son demasiado abundantes (Ramos, Fernández, Rey, Rodríguez, y Ros, 2015), pero la inmensa mayoría destaca diferencias en favor de las mujeres (Kenney, Pomerantz, Tyan, y Patrick, 2006; Kinzie et al., 2007; Lam, Wong, Yang, y Liu, 2012; Marks, 2000). Por el contrario, también existen estudios que afirman no encontrar diferencias significativas en función del sexo dependiendo del instrumento de medida utilizado (Ros, Goikoetxea, Gairín, y Lekue, 2012). Esto podría estar relacionado con el profesorado y su comportamiento ya que hay investigaciones que destacan su influencia en la implicación estudiantil (Wang y Eccles, 2012; Wang y Holcombe, 2010).

Respecto al nivel educativo de las madres y padres, estos son un agente que repercute directamente en la participación escolar de los estudiantes (De León, 2011). Algunos estudios avalan que el nivel educativo parental influye en el desarrollo, desempeño y comportamiento escolar de sus descendientes (Halpern, 1986; Jadue, 2003; Parcel, Dufur, y Cornell, 2010; Razeto, 2016). Otras investigaciones, por el contrario, resaltan el papel de la familia en el desempeño escolar de los estudiantes, pero destacan las ideas, actitudes y actividades de los progenitores frente a sus características concretas como su nivel educativo (Feinstein, Duckworth, y Sabates, 2008). En consecuencia, no existe unanimidad de criterios en cuanto a la influencia que el nivel educativo de los progenitores puede ejercer en una variable como la implicación escolar de los estudiantes.

De este modo, los objetivos de esta investigación serían dos: (a) analizar las relaciones entre el apoyo social percibido y la implicación escolar; (b) identificar la variabilidad del apoyo social percibido y de la implicación escolar en razón de las variables socio-personales sexo y nivel académico de los progenitores.

\section{MÉTODO}

\section{Participantes}

Participaron un total de 331 estudiantes de los cuales se eliminaron 8 sujetos, dos por no responder algún ítem (más de $1 \%$ del total de ítems) y seis por mantener patrones de respuesta extraños o no consistentes. Así, quedaron finalmente 323 estudiantes de E.S.O de entre 13 y 18 años de edad $(M=14.41 ; D T=1.18)$ pertenecientes a aun centro concertado de Álava con un nivel socioeconómico medio-alto. El 60.4\% de las personas participantes eran mujeres y el $39.6 \%$ restante hombres, considerándose grupos equilibrados $\left(\chi^{2}=4.87 ; p>.05\right)$. La selección de la muestra se realizó de forma incidental. 


\section{Instrumentos}

Como variables sociopersonales se incluyen el sexo y el nivel educativo de los progenitores (estudios hasta la E.S.O, formación profesional o universidad). Además, los participantes rellenaron diversos cuestionarios, todos ellos respondidos mediante escala Likert de 5 grados.

Para evaluar el apoyo social percibido se empleó el cuestionario APIK (Izar de la Fuente, 2018) que evalúa tres dimensiones (apoyo familiar, apoyo del profesorado y apoyo de las amistades) y está constituido por 27 ítems con una consistencia interna de $\alpha=.90$ para el presente estudio.

Pala evaluar la implicación escolar se utilizó la versión española del School Engagement Measure (SEM; Fredricks, Blumenfeld, Friedel, y Paris, 2005) validada por Ramos, Rodríguez, y Revuelta (2016). Este instrumento que evalúa tres dimensiones (implicación cognitiva, emocional y conductual) está compuesto por 19 ítems y ha mostrado una consistencia interna de $\alpha=.83$ en este estudio.

\section{Procedimiento}

Se mantuvo una reunión con la dirección del centro educativo y tras la aceptación de participar en la presente investigación se les envió a los padres o tutores legales el consentimiento informado que debían firmar. En dicho consentimiento se informaba de los objetivos de la investigación, de la batería de cuestionarios a aplicar, del uso ético de los datos recogidos y de la posibilidad de rechazar en cualquier momento el uso de los mismos. La administración de los cuestionarios se hizo de manera individual y colectiva dentro del horario lectivo y en las propias aulas por el autor del estudio, controlando previamente que la extensión de la batería de cuestionario no superara los 20 minutos de cumplimentación. El propio investigador se encargó de resolver las dudas que surgían y para evitar sesgos que cuestionaran la validez de los resultados, no se reveló la finalidad de la investigación a los participantes y se aseguró el anonimato junto con la voluntariedad de la participación, a fin de reducir la tendencia de deseabilidad social.

\section{Analisis de datos}

Se eliminaron los sujetos que no habían respondido algún ítem (más del 1\%) y los que mostraron patrones de respuesta extraños mediante la prueba de clusters. Los valores perdidos (menos de 1\%) se reemplazaron con el método de estimación de tendencia lineal en el punto.

Posteriormente se realizó el análisis de normalidad, resultando algunos datos normales y otros no. Esto podría suponer una violación de la normalidad, pero la asimetría y curtosis no se alejan demasiado de la normalidad y además las pruebas paramétricas son suficientemente robustas a dicha violación (Chok, 2010; Edgell y Noon, 1984; Schmider, Ziegler, Danay, Beyer, y Bühner, 2010), por lo que se procede a utilizarlas. 
En el análisis de la variabilidad para la variable sexo se llevó a cabo una comparación de medias simples para dos muestras independientes realizada a través de la prueba $t$ de Student. Dado que en el segundo análisis (nivel académico de los progenitores) se tenían que comparar más de dos categorías (E.S.O., FP y Universidad) se utilizó el análisis de la varianza factorial (ANOVA). Posteriormente, con intención de estudiar las posibles diferencias, se realizaron comparaciones múltiples (post-hoc) de Tukey y/o de Games-Howell dependiendo de la existencia o no de homogeneidad de varianzas.

Para el análisis de las relaciones entre las variables apoyo social percibido e implicación escolar se utilizaron correlaciones de Pearson.

\section{RESULTADOS}

Relaciones entre el apoyo social percibido y la implicación escolar

Para el primer objetivo de la investigación se llevan a cabo análisis de correlaciones (ver tabla 1).

Tabla 1. Correlaciones entre el apoyo social percibido y la implicación escolar

\begin{tabular}{|c|c|c|c|c|c|}
\hline & & Imp. Conductual & Imp. Afectiva & Imp. Cognitiva & Imp. Total \\
\hline \multirow{2}{*}{ Apoyo familia } & Pearson & $.354^{* * *}$ & $.313^{* * * *}$ & $.266^{* * *}$ & $.386^{* * * *}$ \\
\hline & $p$ & .000 & .000 & .000 & .000 \\
\hline \multirow{2}{*}{ Apoyo amigos } & Pearson & .096 & $.265^{* * * *}$ & .096 & $.199^{* * * *}$ \\
\hline & $p$ & .086 & .000 & .084 & .000 \\
\hline \multirow{2}{*}{ Apoyo profesores } & Pearson & $.359^{* * *}$ & $.522^{* * * *}$ & $.428^{* * * *}$ & $.576^{* * *}$ \\
\hline & $p$ & .000 & .000 & .000 & .000 \\
\hline \multirow{2}{*}{ Apoyo total } & Pearson & $.384^{* * *}$ & $.525^{* * * *}$ & $.385^{* * * *}$ & $.558^{* * * *}$ \\
\hline & $p$ & .000 & .000 & .000 & .000 \\
\hline
\end{tabular}

Los resultados indican que el apoyo social percibido total y la implicación escolar total correlacionan significativamente de manera positiva $(r=.558 ; p<.05)$. También existen correlaciones significativas entre todas las dimensiones a excepción del apoyo de los amigos con la implicación conductual ( $r=.096 ; p>.05)$ y con la implicación cognitiva $(r=.096 ; p>.05)$. La intensidad de las correlaciones del apoyo social percibido con la implicación escolar no es igual para todas las fuentes del apoyo. El apoyo del profesorado correlaciona con la mayor intensidad con las tres escalas de la implicación escolar, logrando la mayor asociación con la escala de la implicación afectiva $(r=.522$; $p<.05)$. Seguidamente se sitúa la dimensión de apoyo familiar mostrando la mayor correlación con la implicación conductual ( $r=.354 ; p<.05)$. Por último, el apoyo de los amigos, el menos relacionado con la implicación escolar, únicamente muestra una correlación significativa de baja intensidad con la implicación afectiva $(r=.265 ; p<.01)$. 


\section{Variabilidad del apoyo social percibido}

En la tabla 2 se recogen las diferencias en el apoyo social percibido en función de las variables socio-personales sexo y nivel académico de los progenitores. Para el segundo se han analizado las diferencias de medias únicamente en la dimensión apoyo familiar dado que para el resto carecería de sentido.

Tabla 2. Diferencias en apoyo social percibido según el sexo

\begin{tabular}{|c|c|c|c|c|c|c|}
\hline & & $n$ & $M$ & $S D$ & $t$ & $p$ \\
\hline \multicolumn{7}{|c|}{ Sexo } \\
\hline \multirow{2}{*}{ Apoyo familiar } & Mujer & 195 & \multirow{2}{*}{$\begin{array}{l}39.76 \\
39.95\end{array}$} & 5.87 & \multirow{2}{*}{-.293} & \multirow{2}{*}{.770} \\
\hline & Hombre & 128 & & 5.07 & & \\
\hline \multirow{2}{*}{ Apoyo amistades } & Mujer & 195 & 39.79 & 5.00 & \multirow{2}{*}{3.140} & \multirow{2}{*}{.002} \\
\hline & Hombre & 128 & 37.89 & 5.80 & & \\
\hline \multirow{2}{*}{ Apoyo profesorado } & Mujer & 195 & 31.03 & 7.70 & \multirow{2}{*}{-.470} & \multirow{2}{*}{.639} \\
\hline & Hombre & 128 & 31.43 & 7.20 & & \\
\hline \multirow{2}{*}{ Apoyo total } & Mujer & 195 & 110.59 & 13.95 & \multirow{2}{*}{.858} & \multirow{2}{*}{.392} \\
\hline & Hombre & 128 & 109.27 & 12.83 & & \\
\hline Diferencias en apoy & percibido & egún el & vel acadé & o de las $n$ & ladres y & los pa \\
\hline \multirow{6}{*}{ Apoyo familiar } & \multirow{3}{*}{$\begin{array}{l}\text { Estudios } \\
\text { madre }\end{array}$} & ESO & $\begin{array}{ll}32 & 3 \\
\end{array}$ & $\begin{array}{ll}4 \quad 6.53 \\
\end{array}$ & \multirow{3}{*}{.047} & \multirow{3}{*}{.954} \\
\hline & & FP & 85 & 5.30 & & \\
\hline & & UNI & 206 & 5.55 & & \\
\hline & \multirow{3}{*}{$\begin{array}{l}\text { Estudios } \\
\text { padre }\end{array}$} & ESO & 45 & 6.63 & \multirow{3}{*}{2.245} & \multirow{3}{*}{.108} \\
\hline & & FP & 91 & 5.70 & & \\
\hline & & UNI & 187 & 5.18 & & \\
\hline
\end{tabular}

En lo que respecta al sexo, únicamente existen diferencias significativas en el apoyo social percibido de las amistades $(t=3.14 ; p=.002)$ a favor de las mujeres. En cuanto al nivel de apoyo familiar percibido en función del nivel académico de la madre y del padre, no se han encontrado diferencias significativas para ninguno de los dos casos.

\section{Variabilidad de la implicación escolar}

Para el tercer objetivo de la investigación se realizan comparaciones de medias (ver tabla 3) de la implicación teniendo en cuenta las variables sexo y nivel académico de padres y madres.

Tal y como se puede observar en la tabla, en lo que respecta al sexo y al nivel académico de los progenitores no existen diferencias significativas en la implicación escolar ni en ninguna de sus dimensiones. 
IZAR DE LA FUENTE et al. Apoyo social e implicación escolar

\begin{tabular}{|c|c|c|c|c|c|c|c|}
\hline & & & $\mathrm{n}$ & $M$ & $S D$ & $t$ & $p$ \\
\hline \multicolumn{8}{|c|}{ Sexo } \\
\hline IMPLICACIÓN & \multirow{2}{*}{\multicolumn{2}{|c|}{$\begin{array}{c}\text { Mujer } \\
\text { Hombre }\end{array}$}} & 195 & 21.13 & 2.46 & \multirow{2}{*}{.753} & \multirow{2}{*}{.452} \\
\hline CONDUCTUAL & & & 128 & 20.92 & 2.47 & & \\
\hline IMPLICACIÓN & \multicolumn{2}{|c|}{ Mujer } & 195 & 22.23 & 4.11 & \multirow{2}{*}{1.260} & \multirow{2}{*}{.209} \\
\hline AFECTIVA & \multicolumn{2}{|c|}{ Hombre } & 128 & 21.62 & 4.50 & & \\
\hline IMPLICACIÓN & \multirow{2}{*}{\multicolumn{2}{|c|}{$\begin{array}{c}\text { Mujer } \\
\text { Hombre }\end{array}$}} & 195 & 24.35 & 5.71 & \multirow{2}{*}{1.439} & \multirow{2}{*}{.151} \\
\hline COGNITIVA & & & 128 & 23.42 & 5.64 & & \\
\hline IMPLICACIÓN & \multirow{2}{*}{\multicolumn{2}{|c|}{$\begin{array}{l}\text { Mujer } \\
\text { Hombre }\end{array}$}} & 195 & 67.73 & 9.38 & \multirow[b]{2}{*}{1.603} & \multirow{2}{*}{.110} \\
\hline TOTAL & & & 128 & 65.98 & 9.97 & & \\
\hline \multicolumn{8}{|c|}{ Nivel académico de los padres } \\
\hline \multirow{3}{*}{$\begin{array}{l}\text { IMPLICACIÓN } \\
\text { CONDUCTUAL }\end{array}$} & \multirow{3}{*}{ Estudios padre } & ESO & 45 & 21.65 & 2.29 & \multirow{3}{*}{1.941} & \multirow{3}{*}{.145} \\
\hline & & FP & 91 & 20.76 & 2.63 & & \\
\hline & & UNI & 187 & 21.05 & 2.40 & & \\
\hline \multirow{3}{*}{$\begin{array}{l}\text { IMPLICACIÓN } \\
\text { AFECTIVA }\end{array}$} & \multirow{3}{*}{ Estudios padre } & ESO & 45 & 21.38 & 4.73 & \multirow{3}{*}{.591} & \multirow{3}{*}{.554} \\
\hline & & FP & 91 & 22.21 & 4.59 & & \\
\hline & & UNI & 187 & 22.05 & 4.00 & & \\
\hline \multirow{3}{*}{$\begin{array}{l}\text { IMPLICACIÓN } \\
\text { COGNITIVA }\end{array}$} & \multirow{3}{*}{ Estudios padre } & ESO & 45 & 25.13 & 5.18 & \multirow{3}{*}{1.597} & \multirow{3}{*}{.204} \\
\hline & & FP & 91 & 24.35 & 5.67 & & \\
\hline & & UNI & 187 & 23.56 & 5.81 & & \\
\hline & & ESO & 45 & 68.17 & 9.43 & & \\
\hline IMPLICACION & Estudios padre & $\mathrm{FP}$ & 91 & 67.33 & 9.85 & .481 & .619 \\
\hline & & UNI & 187 & 66.67 & 9.62 & & \\
\hline & & $\overline{\text { el acac }}$ & iico dt & adres & & & \\
\hline & & ESO & 32 & 21.25 & 2.60 & & \\
\hline IMPLICACION & Estudios & $\mathrm{FP}$ & 85 & 20.81 & 2.73 & .600 & .549 \\
\hline & & UNI & 206 & 21.12 & 2.33 & & \\
\hline & & ESO & 32 & 21.11 & 5.51 & & \\
\hline IMPLICACION & Estudios & FP & 85 & 22.39 & 4.17 & 1.026 & .360 \\
\hline & & UNI & 206 & 21.98 & 4.10 & & \\
\hline & & ESO & 32 & 25.12 & 4.88 & & \\
\hline IMPLICACIÓN & Estudios & $\mathrm{FP}$ & 85 & 24.30 & 5.80 & .997 & .370 \\
\hline COGNITIVA & madre & UNI & 206 & 23.70 & 5.77 & & \\
\hline & & ESO & 32 & 67.49 & 9.63 & & \\
\hline IMPLICACION & Estudios & $\mathrm{FP}$ & 85 & 67.51 & 9.58 & .193 & .825 \\
\hline & & UNI & 206 & 66.81 & 9.71 & & \\
\hline
\end{tabular}

\section{DISCUSIÓN Y CONCLUSIONES}

El primer objetivo de esta investigación se centraba en la relación entre el apoyo social percibido y la implicación escolar. Coincidiendo con estudios anteriores (Christenson y Thurlow, 2004; Perdue et al., 2009; Roorda et al., 2011), los resultados indican que el apoyo de la familia, los iguales y el profesorado correlacionan con la implicación escolar. No obstante, del mismo modo que en otros estudios (Wang y Eccles, 2012), no todas las fuentes de apoyo han tenido el mismo efecto, ya que el apoyo del profesorado es el que correlaciona con mayor intensidad con todas las dimensiones de la implicación escolar, seguido del apoyo familiar. En último lugar, mostrando únicamente correlaciones de baja intensidad con la implicación afectiva se sitúa el apoyo de los iguales. Estos datos concuerdan casi en su totalidad con un estudio previo (Ramos, 2015), a excepción de que en la presente investigación, el apoyo familiar ha mostrado su mayor 
correlación con la implicación conductual, en vez de con la afectiva. El motivo principal podría basarse en que la familia puede influir en mayor medida en aspectos conductuales como la asistencia a clase que en otros emocionales que son de carácter más personal (Schaefer, Coyne, y Lazarus, 1981).

Respecto al segundo objetivo, del mismo modo que en anteriores investigaciones, en el apoyo familiar (Musitu y Cava, 2003) y del profesorado (Malecki y Demaray, 2003) no se han encontrado diferencias significativas entre hombres y mujeres. En el apoyo de las amistades, por el contrario, si existen diferencias significativas, siendo éstas a favor de las mujeres, resultado que concuerda con estudios previos (Malecki y Demaray, 2003; Musitu y Cava, 2003). Esta diferencia podría deberse a las diferentes formas de relacionarse que tienen las mujeres y los hombres (Azpiazu, 2016) dado que al estereotipo femenino se le asignan valores como la cercanía y al masculino, en cambio, la independencia.

A diferencia de otras investigaciones (Bazán et al., 2007; Mangrio et al., 2011), en el presente estudio no se han encontrado diferencias significativas en apoyo familiar, ni de la madre ni del padre, en función de su nivel educativo, lo cual indica que los progenitores apoyan a sus hijos independientemente de su nivel de estudios. Ahora bien, no se ha podido analizar si esa ausencia de diferencias se mantendría en familias sin estudios. Estos datos pudieran deberse a que en la concepción teórica del apoyo social el conocimiento académico no ejerce ningún papel relevante.

En la segunda parte del segundo objetivo del estudio se analizaba la variabilidad de la implicación escolar en razón del sexo y, a diferencia de la gran mayoría de investigaciones (Kenney et al., 2006; Kinzie et al., 2007; Lam et al., 2012; Marks, 2000), en este estudio no se han encontrado diferencias significativas. Es posible que sea debido al profesorado y a su comportamiento (Wang y Eccles, 2012; Wang y Holcombe, 2010) dado que este fomenta la implicación de los estudiantes independientemente del sexo de los estudiantes.

Respecto a la variabilidad de la implicación escolar en función del nivel educativo de los progenitores, en contra de otras investigaciones (Halpern, 1986; Jadue, 2003; Parcel et al., 2010; Razeto, 2016), no se han encontrado diferencias significativas. El motivo podría ser que el papel de la familia en el desempeño escolar estudiantil se basa principalmente en las percepciones, acciones y actitudes de esta frente a otras características más personales como su nivel educativo (Feinstein et al., 2008).

Dicho esto, habría que destacar las limitaciones del estudio dado que la participación obtenida se ha limitado a un único centro escolar de nivel sociocultural medio-alto. En consecuencia, los datos podrían variar si se realiza el estudio en centros escolares de niveles más diversos. Por ello, futuras investigaciones deberían repetir el estudio con muestras variadas. 
Finalmente, de cara al futuro se pretende continuar con este estudio investigando más ampliamente las relaciones entre el apoyo social percibido y la implicación escolar sometiendo a prueba un modelo teórico mediante la metodología de modelos de ecuaciones estructurales (SEM) para así poder analizar con mayor detalle el tipo de relación existente entre las variables.

\section{Agradecimientos}

Esta investigación forma parte del trabajo realizado dentro del Grupo Consolidado de Investigación del Sistema Universitario Vasco IT934-16, de los resultados del proyecto de investigación PPG17/61 de la Universidad del País Vasco y del proyecto EDU2017-83949-P del subprograma estatal de Generación del Conocimiento del Ministerio de Economía, Industria y Competitividad. Asimismo, el primer autor del artículo es beneficiario del Programa Predoctoral de Formación de Personal Investigador No Doctor del Departamento de Educación del Gobierno Vasco.

\section{REFERENCIAS}

Alemán, I.L. (2013). Desarrollo y validación del cuestionario de apoyo social V.I.D.A. (vínculos interpersonales de apoyo). Tesis doctoral. Las Palmas de Gran canaria: Universidad de Las Palmas de Gran Canaria.

Azpiazu, L. (2016). El ajuste escolar: Un modelo explicativo en función de variables contextuales y personales. Tesis doctoral. Lejona: UPV/EHU.

Barrón, A. (1996). Apoyo social: Aspectos teóricos y aplicaciones. Madrid: Siglo XXI.

Barrón, A., y Sánchez, E. (2001). Estructura social, apoyo social y salud mental. Psicothema, 13(1), 17-23.

Bazán, A., Sánchez, B., y Castañeda, S. (2007). Relación estructural entre apoyo familiar, nivel educativo de los padres, características del maestro y desempeño en lengua escrita. Revista Mexicana de Investigación educativa, 12(33), 701-729.

Caplan, G. (1974). Support systems and community mental health: Lectures in concept development. New York: Behavioral Publications.

Cassel, J.C. (1974a). Psychiatric epidemiology. En G. Caplan (Ed.), Americans handbook of psychiatry (pp. 401-411). New York: Basic Books.

Cassel, J.C. (1974b). Psychosocial process and "stress": Theoretical formulations. International Journal of Health Services, 4(3), 471-482.

Cheng, S., y Chan, A. (2004). The Multidimensional Scale of Perceived Social Support: Dimensionality and age and gender differences in adolescents. Personality and Individual Differences, 37(7), 1359-1369.

Chok, N.S. (2010) Pearson's versus Spearman's and Kendall's Correlation Coefficients for Continuous Data. Tesis doctoral. Pittsburgh: Universidad de Pittsburgh.

Christenson, S.L., y Thurlow, M.L. (2004). School dropouts: Prevention considerations, interventions, and challenges. Current Directions in Psychological Science, 13, 36-39.

Cobb, S. (1976). Social support as a moderator of life stress. Psychosomatic Medicine, 38(5), 300314. 
Cohen, S., Mermelstein, R., Kamarck, T., y Hoberman H.M. (1985). Measuring the functional components of social support. En I.G. Sarason y B.R. Sarason (Eds.), Social support: Theory, research and applications (pp. 73-94). Dordrecht: Martinus Nijhoff Publishers.

Demaray, M., y Malecki, C., (2002). Critical levels of perceived social support associated with student adjustment. School Psychology Quarterly, 17(3), 213-241.

De León, B. (2011). La relación familia-escuela y su repercusión en la autonomía y responsabilidad de los niños/as. Actas XII Congreso Internacional de Teoría de la Educación, 1-20.

Edgell, S.E., y Noon, S.M. (1984). Effect of violation of normality on the T-test of the correlation coefficient. Psychological Bulletin, 95(3), 576-583.

Feinstein, L., Duckworth, K., y Sabates, R. (2008). Education and the Family. Passing Success across the Fenerations. London: Routledge.

Fredricks, J.A., Blumenfeld, P.C., Friedel, J., y Paris, A. (2005). School engagement. En K.A. Moore y L. Lippman (Eds.), Conceptualizing and measuring indicators of positive development: What do children need to flourish (pp. 305-321). New York: Kluwer Academic/Plenum Press.

Fredricks, J.A., Blumenfeld, P.C., y Paris, A.H. (2004). School engagement: Potential of the concept, state of the evidence. Review of Educational Research, 74(1), 59-109.

Furrer, C., y Skinner, E. (2003). Sense of relatedness as a factor in children's academic engagement and performance. Journal of Educational Psychology, 95(1), 148-162.

Gracia, E., Herrero, J., y Musitu, G. (2002). Evaluación de recursos estresores y psicosociales en la comunidad. Madrid: Síntesis.

Halpern, R. (1986). Effects of Early Childhood Intervention on Primary School Progress in Latin America. Comparative Education Review, 30(2), 193-215.

Izar de la Fuente, I. (2018). Diseño y validación de un instrumento para la medida del apoyo social percibido (APIK) en la adolescencia (Trabajo de fin de máster no publicado). UPV/EHU, Lejona.

Jadue, G. (2003). Transformaciones familiares en Chile: riesgo creciente para el desarrollo emocional, psicosocial y la educación de los hijos. Estudios Pedagógicos, 29, 115-126.

Kenney, G.A., Pomerantz, E.M., Ryan, A.M., y Patrick, H. (2006). Sex differences in math performance: The role of children's approach to schoolwork. Developmental Psychology, 42(1), 11-26.

Kinzie, J., Gonyea, R., Kuh, G.D., Umbach, P., Wabash, C.B., y Korkmaz, A. (2007). The relationship between gender and student engagement in college. Trabajo publicado en la $2^{\text {nd }}$ annual conference of the Association for the Study of Higher Education, Louisville.

Lam, S., Wong, B.H., Yang, H., y Liu, Y. (2012). Understanding student engagement with a contextual model. En S.L. Christenson, A.L. Reschly, y C. Wylie (Eds.), Handbook of research on student engagement (pp. 403-419). New York, NY US: Springer Science.

Lewis, A.D., Huebner, E., Malone, P.S., y Valois, R.F. (2011). Life satisfaction and student engagement in adolescents. Journal of Youth and Adolescence, 40(3), 249-262.

Lin, N. (1986). Conceptualizing social support. En N. Lin, A. Dean, y W. Ensel (Eds.), Social support, life events, and depression (pp. 17-30). New York: Academic Press.

Malecki, C., y Demaray, M. (2003). What type of support do they need? Investigating student adjustment as related to emotional, informational, appraisal, and instrumental support. School Psychology Quarterly, 18(3), 231-252.

Mangrio, E., Hansen, K., Lindstrom, M., Kohler, M., y Rosvall, M. (2011). Maternal educational level, parental preventive behaviour, risk behaviour, social support and medical care consumption in 8-month-old children in Malmo, Sweden. BMC public health, 11(891), 19. 
Marks, H. (2000). Student engagement in instructional activity: Patterns in elementary, middle and high school years. American Educational Research Journal, 37, 153-184.

Musitu, G., y Cava, M.J. (2003). El rol del apoyo social en el ajuste de los adolescentes. Intervención Psicosocial, 12(2), 179-192.

Parcel, T., Dufur, M., y Cornell, R. (2010). Capital at Home and at School: A Review and Synthesis. Journal of Marriage and Family, 72, 828-846.

Perdue, N.H., Manzeske, D.P., y Estell, D.B. (2009). Predicting school commitment at grade five: Exploring the role of students' relationships with peers and teachers from grade three. Psychology in the Schools, 46, 1084-1097.

Ramos, E. (2015). Resiliencia y ajuste psicosocial en la adolescencia. Tesis doctoral. Lejona: UPV/EHU.

Ramos, E., Fernández, A., Rey, A., Rodríguez, A., y Ros, I. (2015). Eskola-inplikazioa: kontzeptua, neurketa, aldakortasuna eta testuinguruaren aldagaiak nahiz aldagai psikologikoak. Tantak, 27(2), 149-168.

Ramos, E., Rodríguez, A., y Revuelta, L. (2016). Validation of the Spanish Version of the School Engagement Measure (SEM). Spanish Journal of Psychology, 19(86), 1-9.

Razeto, A. (2016). El involucramiento de las familias en la educación de los niños. Cuatro reflexiones para fortalecer la relación entre familias y escuelas. Revista Páginas de Educación, 9(2), 184-201.

Reddy, R., Rhodes, J.E., y Mulhall, P. (2003). The influence of teacher support on student adjustment in the middle school years: A latent growth curve study. Development and Psychopathology, 15(1), 119-138.

Rodríguez, A., Ramos, E., Ros, I., Fernández, A., y Revuelta, L. (2016). Bienestar subjetivo en la adolescencia: El papel de la resiliencia, el autoconcepto y el apoyo social percibido. Suma Psicológica, 23(1), 60-69.

Rodríguez, A., Ramos, E., Ros, I., y Zuazagoitia, A. (2018). Implicación escolar de estudiantes de secundaria: La influencia de la resiliencia, el autoconcepto y el apoyo social percibido. Educación XX1, 21(1), 87-108.

Roeser, R.W., Eccles, J.S., y Sameroff, A.J. (2000). School as a context of early adolescents' academic and social-emotional development: A summary of research findings. The Elementary School Journal, 100(5), 443-471.

Roorda, D.L., Koomen, H.M., Spilt, J.L., y Oort, F.J. (2011). The influence of affective teacherstudent relationships on students' school engagement and achievement a metaanalytic approach. Review of Educational Research, 81(4), 493-529.

Ros, I., Goikoetxea, J., Gairín, J., y Lekue, P. (2012). Implicación del alumnado en la escuela: Diferencias interindividuales e intercentros. Revista de Psicodidáctica, 17(2), 291-307.

Ros, I., y Zuazagoitia, A. (2015). Implicación escolar y autoconcepto. En A. Fernández y L. Revuelta (Eds.), Ajuste personal y social. Investigación psicoeducativa (pp. 119-129). San Sebastian: Erein.

Schaefer, C., Coyne, J., y Lazarus, R. (1981). The health-related functions of social support. Journal of Behavioral Medicine, 4(4), 381-406.

Schmider, E., Ziegler, M., Danay, E., Beyer, L., y Bühner, M. (2010). Is it really robust? Reinvestigating the robustness of ANOVA against violations of the normal distribution assumption. Methodology, 6(4), 147-151.

Tardy, C.H. (1985). Social support measurement. American Journal of Community Psychology, 13(2), 187-202.

Veiga, F.H., Burden, R., Appleton, J., Taveira, M., y Galvao, D. (2014). Envolvimiento de los estudiantes en la escuela: conceptualización y relaciones con variables personales y 
rendimiento académico. Una revisión de la literatura. Revista de Psicología y Educación, 9(1), 29-47.

Voelkl, K.E. (2012). School identification. En S. L. Christenson, A. L. Reschly y C. Wylie (Eds.), Handbook of research on student engagement (pp. 193-218). New York: Springer.

Wang, M., y Holcombe, R. (2010). Adolescents' perceptions of school environment, engagement, and academic achievement in middle school. American Educational Research Journal, 47(3), 633-662.

Wang, M., y Eccles, J.S. (2012). Social support matters: Longitudinal effects of social support on three dimensions of school engagement from middle to high school. Child Development, 83(3), 877-895.

Wilcox, B.L., y Vernberg, E.M. (1985). Conceptual and theoretical dilemnas facing social support research. En I. G. Saranson y B. R. Saranson (Eds.), Social support: Theory, Research and Applications (pp. 3-20). Dordrecht: Martinus Nijhoff Publishers.

Recibido: 21 de abril de 2019

Recepción Modificaciones: 30 de abril de 2019

Aceptado: 3 de mayo de 2019 